\title{
STRICT CONVEXITY AND SMOOTHNESS OF NORMED SPACES
}

\author{
BY \\ MAHLON M. DAY
}

1. Definitions and outline of results. This paper contains the first examples of normed spaces not isomorphic to strictly convex or smooth spaces. The table below shows the properties now known to be possessed by a number of special Banach spaces; some conclusions and unsolved problems are discussed after the table. $\$ 2$ contains the positive results which enable us to show that the special examples do have the properties asserted, while $\S 3$ contains the specific calculations which show that some spaces can not be made smooth or strictly convex. $\$ 4$ contains an example related to the impossibility of some generalizations of theorems of Kakutani and Michael on simultaneous extension of continuous functions; the proof presented for this example shows that this topic is, slightly, related to the one discussed in detail here.

The following definitions and notation are used throughout the paper.

Definitions. Let $B$ be a normed linear space. If every chord of the unit sphere of $B$ has its midpoint below the surface of the unit sphere, then $B$ is called strictly convex (written SC); if through every point of the surface of the unit sphere of $B$ there passes a unique hyperplane of support (that is, a tangent hyperplane) of the unit sphere, then $B$ is called smooth (written $\mathrm{SM})$; if both occur, then $B$ is called SCM. If $B$ is isomorphic to an $S X$ space, $X=C, M$, or $C M$, then $B$ is called an $s x$ space.

If $I$ is an index set, let $K=K(I)$ be the Tyhonov cube; that is, the topological product $\mathrm{P}_{i \in I} J_{i}$, where each $J_{i}$ is the closed interval $[-1,1]$. For $p \geqq 1$ let $H_{p}=H_{p}(I)$ be the set of those elements $x$ of $K(I)$ such that

$$
\sum_{i \in I}|x(i)|^{p} \leqq 1
$$

Also define

$m(I)$ space of all bounded real functions on $I$ with $\|x\|=\operatorname{lub}_{i \in I}|x(i)|$.

$m_{0}(I)$ subspace of all those $x$ in $m(I)$ which vanish except on a countable set.

$c_{0}(I)$ subspace of those $x$ in $m(I)$ for which for each $\epsilon>0$ the set of $i$ with $|x(i)|>\epsilon$ is finite; that is, $c_{0}(I)$ is the set of functions vanishing at infinity on the discrete space $I$.

$$
\begin{aligned}
& l_{p}(I) \text { for } p \geqq 1 \text {, the set of those real functions } x \text { on } I \text { for which }\|x\|_{l_{p}} \\
= & {\left[\sum_{i \in I}|x(i)|^{p}\right]^{1 / p}<\infty . }
\end{aligned}
$$

Presented to the Society, June 19, 1954; received by the editors May 14, 1954. 
Given a set $X$, a Borel field $F$ of sets in $X$, and a countably additive, nonnegative set function $\mu$ defined on $F, \mu$ is called finite, or $\sigma$-finite, if there is a set $X_{1}$, or a sequence of sets $X_{j}$, in $F$ each of finite $\mu$-measure such that every set in $F$ is essentially contained on $X_{1}$, or in $\bigcup_{j} X_{j}$. $<\infty$.

$L_{p}(\mu)$ space of measurable functions on $X$ such that $\|f\| L_{p}=\left[\int|f(x)|^{p} d \mu\right]^{1 / p}$

$M(\mu)$ space of essentially bounded measurable functions on $X$ with essential lub for norm.

$C(X)$ (if $X$ is a topological space) is the space of real-valued, bounded, continuous functions on $X$.

In the table below $I_{c}$ and $I_{u}$ represent countably infinite and uncountable index sets. $\phi$ is a finite or $\sigma$-finite measure sufficiently nontrivial that $M(\phi)$ is not finite-dimensional; $\nu$ is a finite or $\sigma$-finite nonseparable measure; $\lambda$ is a non- $\sigma$-finite measure. $A+(\mathrm{a}-)$ in the table means that the space in that row has (has not) the property in that column; a blank means that the precise position is not yet known.

$\begin{array}{lccccc} & \text { scm } & \text { sc and } & \text { sc, not } & \text { sm, not } & \text { not sc } \\ B \text { separable } & & \text { sm } & \text { sm } & \text { sc } & \text { not sm } \\ c_{0}\left(I_{u}\right) & + & + & - & - & - \\ l_{1}\left(I_{u}\right) & & + & - & - & - \\ m_{0}\left(I_{c}\right) & - & - & + & - & - \\ m_{0}\left(I_{u}\right) & - & - & + & - & - \\ L_{1}(\nu) & - & - & - & - & + \\ L_{1}(\lambda) & - & & - & & - \\ M(\phi) & - & - & & - & \\ M(\lambda) & - & - & + & - & - \\ l_{p}(I) \text { and } L_{p}(\mu), p>1 & + & - & - & - & + \\ C\left(K\left(I_{u}\right)\right) & + & + & - & - & - \\ & - & - & + & - & -\end{array}$

From the above examples and from the results stated more fully in the next section some questions are answered and some new questions made pertinent.

(i) There is a Banach space, $c_{0}\left(I_{c}\right)$, which is scm; its conjugate space (equivalent to $l_{1}\left(I_{c}\right)$ ) is scm; its second conjugate (equivalent to $m\left(I_{c}\right)$ ) is sc, not sm; its third conjugate is not sm, and whether or not sc is mixed up with (v) below.

(ii) There is a Banach space $c_{0}\left(I_{u}\right)$ which is sc and sm; its conjugate space is sc but not sm; its second conjugate space is neither.

For reflexive spaces we have full duality between SC and SM, and also between sc and sm. The $L_{p}(\mu), p>1$, are examples of SCM reflexive spaces. 
(iii) There is no example known of a reflexive space which is not sc or of such a space not sm. Klee has conjectured that perhaps every reflexive space is sc (hence by duality sm); this property, if verified, could be used with weak compactness of spheres as a substitute for uniform convexity in many arguments. It was so used, for example, in a generalization of one of the ergodic theorems of Alaoglu-Birkhoff. (Day, Bull. Amer. Math. Soc. (1941) pp. 313317.)

For a single nonreflexive $B$ there are examples which are scm, or nonseparable but sc and sm, or sc but not sm, or neither sc nor sm.

(iv) There is no example known to be sm but not sc.

The most likely present candidate is an $L_{1}(\nu)$ space; by Theorem 6 we have

(v) If every $L_{1}(\phi)$ is sc, then every $L_{1}(\mu)$ is sc. Restating this in terms of the abstract $L$-spaces of Kakutani, Ann. of Math. vol. 42 (1941) pp. 523-537.

(vi) Every $(A L)$ space is sc if and only if every $(A L)$ space with unit is sc.

(vii) There is no example known of a nonreflexive nonseparable scm space.

For a pair of spaces $B$ and $B^{*}$ in the nonreflexive case there are examples with $B$ and $B^{*}$ both $\mathrm{scm}$, or $B \mathrm{scm}$ and $B^{*} \mathrm{sc}$ but not sm, or $B \mathrm{sc}$ but not sm and $B^{*}$ neither.

(viii) There is no example known with $B$ sm and $B^{*}$ not sc.

2. Some positive results. In the notation of Klee [4], if $S$ stands for one of the two properties SC or SM, let $S^{*}$ stand for the other. It is known (Alaoglu-Birkhoff $[1$, Footnote 13], or Klee [4, A1.1]) that

(1) If $B$ is not $S$, then $B^{*}$ is not $S^{*}$; that is,

$\left(1^{\prime}\right)$ If $B^{*}$ is $S^{*}$, then $B$ is $S$.

For reflexive spaces this means that the properties SC and SM are truly dual to each other; since an isomorphism of either $B$ or $B^{*}$ determines an isomorphism of the other (in this reflexive case) it follows that in reflexive spaces the properties sc and sm are also dual. For nonreflexive spaces an isomorphism of $B^{*}$ may not be determined by an isomorphism of $B$, but Klee has observed (A1.2) that

(2) If $B^{*}$ is $s^{*}$ and if the new unit sphere is $w^{*}$-closed, then $B$ is $s$.

In later applications it will be convenient to rephrase (2) as

$\left(2^{\prime}\right)$ If $B^{*}$ is $s^{*}$ and if the new norm in $B^{*}$ is $w^{*}$-lower semi-continuous and $S^{*}$, then it is the conjugate norm of a new norm in $B$ which is $S$.

Clarkson [2] showed that every separable normed space is sc. His proof uses only the existence of a bounded countable total set of linear functionals $\left\{f_{j}\right\}$ of norm 1 ; mapping $B$ into $l_{2}$ by setting $T x=\left\{f_{j}(x) / 2^{j}\right\}, j=1,2, \cdots$, we see that $T$ is one-to-one and linear from $B$ into the scm space $l_{2}$; the rest of Clarkson's proof can be formalized as of proof of (Klee A1.4(v)).

(3) If and only if there is a one-to-one and linear map of $B$ into an SC (or even sc) space $B^{\prime}$, then $B$ is sc.

The identity map of $l_{1}(I)$ into $l_{2}(I)$ combined with Theorem 7 shows that no such result can hold for smoothness. The best of which I am now aware is 
THEOREM 1. If $B$ is isomorphic with a subspace of a space with $S$ (or $s$ ), then $B$ is $s$.

It suffices to prove that if $B$ is a subspace of $B^{\prime}$ and if $B^{\prime}$ is $S$, so is $B$. For SC this is trivial since all calculations take place in one plane at a time. If $B$ is not $\mathrm{SM}$, there is a point $x \in B$ and two elements $f_{1}$ and $f_{2}$ in $B^{*}$ of norm one such that $f_{1}(x)=\|x\|=f_{2}(x)$. By the Hahn-Banach theorem each $f_{i}$ has an extension $\phi_{i}$ of norm 1 in $B^{\prime *}$; hence $B^{\prime}$ is not smooth if $B$ is not.

A related result is

THEOREM 2. If $B$ is an $S M$ (or even sm) space and if there is a one-to-one linear $T$ from $B$ into an scm space $B^{\prime}$, then $B$ is scm.

We may assume that $B$ is SM and that $B^{\prime}$ is SCM ; then let $p(x)=\|T x\|_{B^{\prime}}$ and let $|x|=\|x\|+p(x)$. If $B^{\prime \prime}$ is $B$ renormed with $|\cdots|$, we see that $B^{\prime \prime}$ is sc (by the proof of (3)). But by the Hahn-Banach theorem smoothness is equivalent to differentiability of the norm in every plane section through 0 . $T$ is an isomorphism in every such plane, so the functions $\|\cdots\|$ and $p(\cdots)$ are both differentiable in every such plane; the same is true of their sum.

We also need a well-known fact:

(4) The norm in a conjugate space is a lower semi-continuous functional when the space is given its $w^{*}$-topology; that is, if $w^{*}-\lim _{a} f_{a}=f$, then $\lim \inf _{a}\left\|f_{a}\right\| \geqq\|f\|$.

THEOREM 3. Let $\left\{x_{i}\right\}$ be a sequence of elements of the unit sphere of a normed space $B$, and for each $f \in B^{*}$ let $T f=\left\{f\left(x_{i}\right) / 2^{i}\right\}$. Then $T\left(B^{*}\right) \subseteq l_{2}$ and

(a) $T$ is continuous if the $w^{*}$-topology is used in $B^{*}$ and coordinatewise convergence is used in $l_{2}$.

(b) If $p(f)=\|T f\|_{l_{2}}$, then $p$ is w*-lower semi-continuous.

(c) If $|f|=\|f\|+p(f)$, then $|\cdots|$ is w*-lower semi-continuous, so $U^{\prime}=\{f|| f \mid \leqq 1\}$ is $w^{*}$ closed in $B^{*}$.

(a) is trivial; (b) follows from (a) and the simple calculation that if $x_{i}^{n} \in l_{2}$ and $\lim _{n} x_{i}^{n}=x_{i}$ for each $i$, then $\sum_{i}\left|x_{i}\right|^{2} \leqq \lim \inf _{n} \sum_{i}\left|x_{i}^{n}\right|^{2}$. (c) follows from (b) and (3), as the sum of lower semi-continuous functions has the same property.

Clarkson [2] showed that every separable space is sc; Klee [4, A1.11] showed from this and (2) that every separable reflexive space is sc and is sm. The next result improves both of these.

Theorem 4. If $B$ is separable, then $B$ is scm.

If $B$ is separable, take the sequence $\left\{x_{j}\right\}$ of Theorem 3 to be dense on the unit sphere of $B$. Then (Clarkson's result or (3)) $B^{*}$ is SC under the new norm $|\cdots|$. But the new unit sphere is $w^{*}$-closed; by $\left(2^{\prime}\right)$ or (2) $B$ is SM under a new norm which we also denote by $|\cdots|$. Now let $\left\{f_{j}\right\}$ be a 
bounded sequence of elements of $B^{*}$ total over $B$, and let $V x=\left\{f_{j}(x) / 2^{j}\right\}$. Then $V$ is one-to-one and linear from $B$ into the SCM space $l_{2}$; by Theorem 2, $B$ is scm.

The next theorem gives our most powerful smoothability proof; Klee (A1.7) has a related result with a stronger conclusion, but his hypotheses are so much stronger that they apply only to reflexive spaces; we wish to apply Theorem 5 to the spaces $c_{0}(I)$ and $L_{1}(\mu)$.

TheOREM 5. Let $B_{0}$ be a reflexive $S M$ space and let $T$ be a linear function carrying $B_{0}$ into a dense subset of $B$. Then $B$ is sm and $B^{*}$ is sc; more specifically,

(a) $T^{*}$ is one-to-one linear between $B^{*}$ and a subspace of $B_{0}^{*}$, and is $w^{*}$-w* continuous.

(b) If $p(f)=\left\|T^{*} f\right\|_{B_{0} *}$, then $p$ is a bounded, sub-additive, positive-homogeneous, w*-lower semi-continuous functional on $B^{*}$.

(c) if $|f|=\|f\|_{B^{*}}+p(f)$, then $|\cdots|$ is an $S C$ norm which is isomorphic to $\|\cdots\|$ in $B^{*}$ and is a conjugate norm.

(d) If $|\cdots|$ is the norm in $B$ whose conjutate in $B^{*}$ is $|\cdots|$, then $|\ldots|$ is $S M$ and is isomorphic to ||$.||$.

Since $T\left(B_{0}\right)$ is dense, $T^{*} f=0$ if and only if $f=0$, so $T^{*}$ is one-to-one into $B_{0}^{*}$. Every adjoint operation is $w^{*}$ - $w^{*}$ continuous. $p$ is the composition of a lower semi-continuous functional on a continuous function, so is lower semicontinuous. The algebraic properties of $p$ and $|\cdots|$ are easily verified. $|\cdots|$ the sum of two lower semi-continuous functionals, is lower semicontinuous: by $\left(2^{\prime}\right),|\cdots|$ is a conjugate norm. $\left(1^{\prime}\right)$ and reflexivity of $B_{0}$ assert $B^{*}$ is SC. By (3), $|\cdots|$ is SC in $B^{*}$. $\left(2^{\prime}\right)$ asserts that $B$ is sm.

In the next theorem possible choices for $F$ are the $l_{p}(I), p>1$. Let $F$ be a normed linear space of real-valued functions $\phi$ on $I$ such that $0 \leqq \phi(i) \leqq \psi(i)$ for all $i$ imply (i) $0 \leqq\|\phi\| \leqq\|\psi\|$. For each $i$ in $I$ let $B_{i}$ be a normed space and let $B$ be a linear space of functions defined on $I$ with $f(i)$ in $B_{i}$ for each $i$ and (ii) defining $f^{\prime}$ by $f^{\prime}(i)=\|f(i)\|$, each $f$ in $B$ determines an $f^{\prime}$ in $F$. Define $\|f\|_{B}=\left\|f^{\prime}\right\|_{F}$.

TheOREM 6. $B$ is strictly convex if $F$ and all $B_{i}$ are.

Suppose $\|f\|=\|g\|=1$ and $\|f+g\|=2$, then $\|f\|+\|g\|=\|f+g\|=\left\|(f+g)^{\prime}\right\|$ $\leqq\left\|f^{\prime}+g^{\prime}\right\| \leqq\left\|f^{\prime}\right\|+\left\|g^{\prime}\right\|=\|f\|+\|g\|$. The equality which this forces at the second $\leqq$ and $F$ SC force $f^{\prime}=g^{\prime}$. Then $(f+g)^{\prime}(i)=f^{\prime}(i)+g^{\prime}(i)$ for all $i$ by strict convexity of $F$; strict convexity of $B_{i}$ says $f(i)=g(i)$.

Corollary. Suppose all $B_{i}$ are sc. Then for $p \geqq 1$ the $l_{p}(I)$ product of the $B_{i}$ is $s c$; for I countable the $m(I)$ product of the $B_{i}$ is sc. If $B$ is sc, then the space of continuous functions from $K(I)$ into $B$ is sc.

Various theorems of the next section assert that SC norms can be introduced in these choices for $F$. 
Theorem 7. If $F$ and $B, F^{*}$ and $B^{*}$ satisfy the conditions before Theorem 6, if for $x$ in $B$ and $f$ in $B^{*} f(x)=\sum_{i \in I} f_{i}\left(x_{i}\right)$, and similarly with $x^{\prime}$ in $F$ and $f^{\prime}$ in $F^{*}$, then $B$ is smooth if $F$ and all $B_{i}$ are smooth.

$B$ not smooth would mean that there exist $x, f$, and $g \neq f$ such that

$$
1=\|x\|=f(x)=g(x)=\|f\|=\|g\| \text {. }
$$

Then

$$
\begin{aligned}
1 & =f(x)=\sum_{i} f_{i}\left(x_{i}\right) \leqq \sum_{i}\left|f_{i}\left(x_{i}\right)\right| \leqq \sum_{i}\left\|f_{i}\right\| \cdot\left\|x_{i}\right\| \\
& =f^{\prime}\left(x^{\prime}\right) \leqq\left\|f^{\prime}\right\| \cdot\left\|x^{\prime}\right\|=1,
\end{aligned}
$$

and similarly for $g(x)$. Hence $f_{i}\left(x_{i}\right)=\left\|f_{i}\right\|\left\|x_{i}\right\|$ and $g_{i}\left(x_{i}\right)=\left\|g_{i}\right\|\left\|x_{i}\right\|$ for all $i$. If $F$ is smooth $f^{\prime}$ and $g^{\prime}$ must be equal for they both define hyperplanes of support to the unit sphere at $b^{\prime}$. Also $f_{i}$ and $g_{i}$ are zero whenever $x_{i}$ is zero, for otherwise by suppressing the extra nonzero terms a new $f$ or $g$ could be found with the same value of $f^{\prime}\left(x^{\prime}\right)$, thus denying sm in $F$. Then if $f_{i}\left(x_{i}\right)$ is not zero, $f_{i}$ and $g_{i}$ both define planes of support at $x_{i}$; if $B_{i}$ is SM, then $f_{i}=g_{i}$. This makes $f=g$, contradicting the original assumption that $f \neq g$. Hence $B$ is SM.

CoROLlaRy. If all $B_{i}$ are sm and if $I$ is countable or if $p>1$, then the $l_{p}(I)$ product of the $B_{i}$ is $s m$.

Allowing for sets of measure zero and replacing sums by integrals, the above theorems apply equally well to the spaces $L_{p}(\mu, B)$ of Bochner measurable functions with $p$ th power summable when $p>1$, or, in the second case, when $p=1$ and the measure is finite.

3. Properties of particular spaces. We give now the first examples of spaces shown not to be sc or sm.

THEOREM 8. Let I be uncountable; then $m_{0}(I)$ is not sc.

Corollary. If $I$ is uncountable, $m(I)$ is not sc.

Phillips [6] announced this result in a footnote added in proofreading; to my knowledge no proof has been published heretofore.

To prove the theorem let $U$ be the unit sphere in $m_{0}(I)$. For an $x$ of norm 1 define $F_{x}$, the facet of $U$ determined by $x$, to be the set of all $y$ in $U$ such that $y(i)=x(i)$ at every point where $x(i) \neq 0$.

If $|\cdots|$ is a new norm isomorphic to the usual norm in $m_{0}(I)$, then the scale can be adjusted so that $\|x\| \leqq|x| \leqq k\|x\|$ for some $k \geqq 1$ and all $x$. Then define

$$
M_{x}=\sup \left\{|y| \mid y \in F_{x}\right\} \text { and } m_{x}=\inf \left\{|y| \mid y \in F_{x}\right\} .
$$

We prove first 


$$
M_{x}+m_{x} \geqq 2|x| \text {. }
$$

Take $\epsilon>0$ and $y \in F_{x}$ such that $|y|<m_{x}+\epsilon$. Then $\|x \pm(y-x)\|=1$ so $x \pm(y-x) \in F_{x}$ and

$$
\begin{aligned}
2|x| & =|2 x|=|x+(y-x)+x-(y-x)| \\
& \leqq|x+(y-x)|+|x-(y-x)|<m_{x}+\epsilon+M_{x} .
\end{aligned}
$$

Letting $\epsilon$ tend to 0 gives $\left(^{*}\right)$ which we rewrite as

$$
m_{x} \geqq 2|x|-M_{x} \text {. }
$$

Take $x_{1}$ so that $\left|x_{1}\right| \geqq(3 k+1) / 4$. Then $m_{x_{1}} \geqq(3 k+1) / 2-k=(k+1) / 2$, so

$$
M_{x_{1}}-m_{x_{1}} \leqq(k-1) / 2 \text {. }
$$

Then take $x_{2}$ in $F_{x_{1}}$ so that $\left|x_{2}\right| \geqq\left(3 M_{x_{1}}+\left|x_{1}\right|\right) / 4$; then $m_{x_{2}} \geqq\left(M_{x_{1}}\right.$ $\left.+\left|x_{1}\right|\right) / 2$, so

$$
M_{x_{2}}-m_{x_{2}} \leqq\left(M_{x_{1}}-\left|x_{1}\right|\right) / 2 \leqq\left(M_{x_{1}}-m_{x_{1}}\right) / 2 \leqq(k-1) / 2^{2} .
$$

An induction process giving $x_{n+1}$ from $x_{n}$ just as $x_{2}$ came from $x_{1}$ yields a sequence of points of $m_{0}(I)$ such that $x_{n+1} \in F_{x_{n}}$ for all $n$ and $M_{x_{n}}-m_{x_{n}}$ $\leqq(k-1) / 2^{n}$. Hence the sequences $M_{x_{n}}$ and $m_{x_{n}}$ have the same limit $\mu$. If $y \in \bigcap_{n} F_{x_{n}}$, then $M_{x_{n}} \geqq|y| \geqq m_{x_{n}}$ for all $n$, so $|y|=\mu$.

Now define $x \in m_{0}(I)$ by the rule: If there is an $n$ such that $x_{n}(i) \neq 0$, let $x(i)=x_{n}(i)$; if all $x_{n}(i)=0$, let $x(i)=0$. Then $y \in F_{x}$ if and only if $y \in \bigcap_{n} F_{x_{n}}$, so $|x|=|y|=\mu$ for all $y$ in $F_{x}$. But $F_{x}$ has on it many line segments of length 2 , so the sphere $|z|=\mu$ is not strictly convex. Hence $m_{0}(I)$ is not sc if $I$ is uncountable.

The corollary follows at once from this theorem and Theorem 1.

THEOREM 9. If $I$ is uncountable, then $l_{1}(I)$ is not sm. If $I$ is infinite, then $m_{0}(I)$ is not sm, so $m(I)$ is not sm.

For a contradiction assume that $\|x\| \geqq|x| \geqq m\|x\|$, and also suppose that $|\cdots|$ is a smooth norm.

If $\|x\|=1$, define $E_{x}=\{y \mid\|y\|=1$ and $|y| \leqq|x|-m\|x-y\| / 4\}$; then

(a) $E_{x}$ is closed.

For the inequalities determining $E_{x}$ contain only continuous functions.

(b) If $y \in E_{x}$, then $E_{y} \subseteq E_{x}$.

If $\|x\|=\|y\|=\|z\|=1$, if $|y| \leqq|x|-m\|x-y\| / 4$ and $|z| \leqq|y|-m\|y-z\| / 4$ then $|z| \leqq|x|-m(|x-y| \mid+\|y-z\|) / 4 \leqq|x|-m\|x-z\| / 4$.

(c) If points $z_{n}$ of norm one are defined so that for each $n z_{n+1} \in E_{z_{n}}$, then $\lim _{n} z_{n}$ exists, call it $z$, and $z$ is in all $E_{z_{n}}$.

For each $n, m \leqq\left|z_{n+1}\right| \leqq\left|z_{n}\right|$ so $\lim _{n}\left|z_{n}\right|=\mu$ exists. But when $\left|z_{n}\right|<\mu+\epsilon$ and $k>n$, then $\mu \leqq\left|z_{k}\right| \leqq\left|z_{n}\right|<\mu+\epsilon$, so ||$z_{k}-z_{n} \|<4 \epsilon / m$. By completeness of 
$l_{1}(I)$ or $m_{0}(I), z_{n}$ converges to some limit $z$. (a) asserts that $z \in E_{z_{n}}$ for all $n$.

Now consider $l_{1}(I)$ in particular and take $\|x\|=1$.

(d) If $\mu_{x}=$ inf $\left\{|y| \mid y \in E_{x}\right\}$, then $\mu_{x}<|x|$.

This depends on

(e) There is $z$ such that $\left\|t z+t^{\prime} x\right\|=|t|+\left|t^{\prime}\right|$.

$\{i \mid x(i) \neq 0\}$ is not all of $I$; take $z$ of norm one so that $z(i)=0$ if $x(i) \neq 0$.

If $C$ is the curve $\{v|| v|=| x \mid\}$ in the plane of $z$ and $x$, smoothness of $|\ldots|$ (and the Hahn-Banach theorem) imply that $C$ has a tangent line at $x$, so for some small arc on one side or the other of $x$ the curve $C$ lies outside the parallelogram with corners at $\pm x$ and $\pm 2 z$. Changing from $z$ to $-z$ if necessary, we have for some small positive $t$ a point $y=t z+(1-t) x$ for which $|y|<|x|(1-t / 2) \leqq|x|-m t / 2=|x|-m \| x-y|| / 4<|x|$. Hence $\mu_{x} \leqq|y|$ $<|x|$.

Now take $\| x_{1}||=1$ and let $\epsilon=\left|x_{1}\right|-\mu_{x_{1}}$. Take $x_{2}$ in $E_{x_{1}}$ within $\epsilon / 4$ of $\mu_{x_{1}} ; \cdots$; take $x_{n+1}$ in $E_{x_{n}}$ within $\epsilon / 4^{n}$ of $\mu_{x_{n}} ; \cdots$. Then $x_{n}$ converges to a point $x$, by (c), and $\mu_{x} \geqq \lim _{n} \mu_{x_{n}}=\lim _{n}\left|x_{n}\right|=|x|$. This contradicts (d), so $l_{1}(I)$ is not sm if $I$ is uncountable.

For $m_{0}(I)$ our proof can also be regarded as a proof that $m_{0}(I) / c_{0}(I)$ is not sm if $I$ is infinite. More specifically, let $F=\left\{y \mid y \in m_{0}(I)\right.$ and for every $\left.z \in c_{0}(I)\|z+y\| \geqq\|y\|=1\right\}$. Then

(f) $F$ is closed in $m_{0}(I)$.

For consider a sequence $\left\{y_{n}\right\}$ in $F$ which converges to a point $y$; take $z$ in $c_{0}(I)$. Then $\|y+z\|=\lim _{n}\left\|y_{n}+z\right\| \geqq \lim _{n}\left\|y_{n}\right\|=\|y\|$.

If we set $F_{x}=E_{x} \cap F$, then the properties (a), (b), and (c) follow for $F_{x}$ from the corresponding properties of $E_{x}$. (d) must be replaced by:

(d') If $\mu_{x}^{\prime}=\inf \left\{|y| \mid y \in F_{x}\right\}$, and if $x \in F$, then $\mu_{x}^{\prime}<|x|$.

The proof will proceed like that for $l_{1}(I)$ as soon as it is proved that for $x$ in $F$ there is a $z$ such that (e) holds and the plane of $z$ and $x$ cuts the sphere $\{x \mid\|x\|=1\}$ in a curve containing only points of $F$. To prove this observe that

(g) $x \in F$ if and only if there is a sequence $\left\{i_{j}\right\}$ of distinct elements of $I$ such that $\lim _{j}\left|x\left(i_{j}\right)\right|=\|x\|=1$.

Once $(\mathrm{g})$ is verified, take $z\left(i_{2 j}\right)=-x\left(i_{2 j}\right)$ for all $j$ and take $z(i)=x(i)$ for all other $i \in I$. Then for $y=t z+t^{\prime} x$, we have $y\left(i_{2 j}\right)=\left(t+t^{\prime}\right) x\left(i_{2 j}\right)$ and $y\left(i_{2 j+1}\right)$ $=\left(t^{\prime}-t\right) x\left(i_{2 j+1}\right)$, so $\|y\|=|t|+\left|t^{\prime}\right| . y$ also satisfies $(\mathrm{g})$ when $|t|+\left|t^{\prime}\right|=1$, so $y \in F$. Starting from a point of $F$ the rest of the proof of $\left(\mathrm{d}^{\prime}\right)$ goes as it did for (d), but working always in $F$. The contradiction derived from smoothness therefore occurs in $m_{0}(I)$ if $I$ is not finite.

THEOREM 10. $c_{0}(I)$ is sc and is sm.

To prove that $c_{0}(I)$ is sc we define a sort of "decreasing rearrangement" map of $c_{0}(I)$ into $l_{2}(I)$; unfortunately it is not linear. Given $x_{1} \in c_{0}(I)$, let $\xi_{1}=\left\|x_{1}\right\|$, and let $E_{1}=\left\{i|| x_{1}(i) \mid=\xi_{1}\right\}$, and let $x_{2}=0$ in $E_{1},=x_{1}$ elsewhere in $I$; 
repeat the process on $x_{2}$, setting $\xi_{2}=\left\|x_{2}\right\|, E_{2}=\left\{i|| x_{2}(i) \mid=\xi_{2}\right.$, and $x_{3}=0$ in $E_{2}, x_{3}=x_{2}$ elsewhere. Continue by induction. Then each $E_{n}$ is a finite set and $E=\mathrm{U}_{n} E_{n}$ can be enumerated in a sequence $\left\{i_{j}\right\}$ in such a way that $\left|x\left(i_{j+1}\right)\right|$ $\leqq\left|x\left(i_{j}\right)\right|$ for all $j$; that is, so that $i_{j}$ in $E_{n}$ and $i_{k}$ in $E_{n+1}$ imply $k>j$. Also $x(i)=0$ if $i \notin E$. We define $D x$ to be the element of $l_{2}(I)$ such that

$$
\begin{aligned}
D x\left(i_{j}\right) & =x\left(i_{j}\right) / 2^{i}, & & \text { for } j=1,2, \cdots, \\
D x(i) & =0 & & \text { for all other } i \in I .
\end{aligned}
$$

Then $D$ is a homogeneous operation from $c_{0}(I)$ into $l_{2}(I)$.

Lemma 1. Let $p(x)=\|D x\|_{l_{2}}$; then $p(a x)=|a| p(x), p(x+y) \leqq p(x)+p(y)$, and $p(x) \leqq\|x\|_{c_{0}}$, so $p$ is continuous.

Since $D(a x)=a D(x)$, the first condition is clear. The last is a consequence of the relation $\sum_{j} 2^{-2 j} \leqq 1$.

To prove subadditivity, take $x, y \in c_{0}(I)$ and suppose that $\left\{i_{j}\right\}$ is a sequence properly chosen for $x+y$, so that

$$
p(x+y)=\left\{\sum_{j}\left[\left(x\left(i_{j}\right)+y\left(i_{j}\right)\right) / 2^{i}\right]^{2}\right\}^{1 / 2} .
$$

By Minkowski's inequality

$$
p(x+y) \leqq\left\{\sum_{j}\left[x\left(i_{j}\right) / 2^{j}\right]^{2}\right\}^{1 / 2}+\left\{\sum_{j}\left[y\left(i_{j}\right) / 2^{j}\right]^{2}\right\}^{1 / 2},
$$

and the proof will be complete if it is shown that, for example,

$$
p(x) \geqq\left\{\sum_{i}\left[x\left(i_{i}\right) / 2^{i}\right]^{2}\right\}^{1 / 2}
$$

Suppose that $\left\{r_{k}\right\}$ is a proper sequence for $x$, so $p(x)=\left\{\sum_{k}\left[x\left(r_{k}\right) / 2^{k}\right]^{2}\right\}^{1 / 2}$; setting $a_{j}=x\left(i_{j}\right)$ and $b_{k}=x\left(r_{k}\right)$ and squaring, we want to show that

$$
\sum_{j}\left(a_{j} / 2^{j}\right)^{2} \leqq \sum_{k}\left(b_{k} / 2^{k}\right)^{2} .
$$

If $m<n$ and also $\left|a_{m}\right|<\left|a_{n}\right|$, then

$$
\begin{aligned}
\frac{a_{m}^{2}}{2^{2 m}}+\frac{a_{n}^{2}}{2^{2 n}} & =2^{-2 n}\left[2^{2 n-2 m} a_{m}^{2}+a_{n}^{2}\right]=2^{-2 n}\left[a_{m}^{2}+a_{n}^{2}+2^{2 n-2 m-1} a_{m}^{2}\right] \\
& >2^{-2 n}\left[a_{m}^{2}+a_{n}^{2}+2^{2 n-2 m-1} a_{n}^{2}\right]=2^{-2 n}\left[2^{2 n-2 m} a_{n}^{2}+a_{m}^{2}\right] \\
& =\frac{a_{n}^{2}}{2^{2 m}}+\frac{a_{m}^{2}}{2^{2 n}},
\end{aligned}
$$

so a permutation of $a_{m}$ and $a_{n}$ increases the left-hand side. Hence we may per- 
mute the $a_{j}$ into a nonincreasing sequence $\left\{c_{n}\right\}$ and get

$$
\sum_{j}\left(a_{j} / 2^{j}\right)^{2} \leqq \sum_{n}\left(c_{n} / 2^{n}\right)^{2}
$$

equality holds at this step if and only if the sequence $\left\{a_{j}\right\}$ is nonincreasing. But the $a_{j}$ which are not zero are some or all of the $b_{k}$. Hence $c_{n} \leqq b_{n}$ for every $n$, so

$$
\sum_{n}\left(c_{n} / 2^{n}\right)^{2} \leqq \sum_{n}\left(b_{n} / 2^{n}\right)^{2} ;
$$

equality holds here if and only if $c_{n}=b_{n}$ for all $n$; that is, if and only if the $a_{j}$ are a permutation of the $b_{n}$.

Proof of Theorem 10. By Theorem $5 l_{1}(I)$ is sc and $c_{0}(I)$ is sm if $T x=x$ is defined from $l_{2}(I)$ in to $c_{0}(I)$.

If $p$ is the function of Lemma 4 and if $|x|=\|x\|+p(x)$, the new norm is isomorphic to the old. To prove it strictly convex, suppose $|x|=|y|=1$ and $|x+y|=|x|+|y|$; then $\|x+y\|=\|x\|+\|y\|$ and $p(x+y)=p(x)+p(y)$. From the well-known conditions for equality in Minkowski's inequality, it follows that if $\left\{i_{j}\right\}$ is a properly chosen sequence for $x+y$, then $x\left(i_{j}\right)=y\left(i_{j}\right)$ for all $j$. But $p(x)>\left\{\sum_{j}\left[x\left(i_{j}\right) / 2^{j}\right]^{2}\right\}^{1 / 2}$ unless $\left\{\left|x\left(i_{j}\right)\right|\right\}$ is already a nonincreasing sequence and $x(i)=0$ if $i$ is not an $i_{j}$. A similar structure holds for $y$, so $x=y$.

Unfortunately, the norm thus constructed in $c_{0}(I)$ is not smooth. For example, if $x=(1,0,0, \cdots)$ and $y=(0,1,0, \cdots)$, then in the plane of $x$ and $y$ the set $\{v \mid p(v) \leqq 1\}$ consists of the common part of the ellipse whose axes end at $\pm 2 x$ and $\pm 4 y$ and the ellipse whose axes end at $\pm 4 x$ and $\pm 2 y$. In the direction of $x+y$, this figure has a corner, so $p$ is not differentiable there.

Theorem 11. If $\mu$ is finite, or even $\sigma$-finite, then $L_{1}(\mu)$ is sm and $M(\mu)$ is sc. If $\mu$ is not $\sigma$-finite, then both these conditions fail.

If $\mu$ is finite then $L_{2}(\mu) \subseteq L_{1}(\mu)$, so the identity mapping of $L_{2}(\mu)$ into $L_{1}(\mu)$ satisfies the hypotheses of Theorem 5. If essentially $X=\bigcup_{j} X_{j}, \mu\left(X_{j}\right)<\infty$, let $\phi_{j}$ be the characteristic function of $X_{j}$ and define $T x=\sum_{j} x \cdot \phi_{j} / 2^{i} \mu\left(X_{j}\right)$. Then $T$ is additive and $\|T x\|_{L_{1}} \leqq\|x\|_{L_{2}}$; also the range of $T$ is dense in $L_{1}(\mu)$, so Theorem 5 applies again. $M(\mu)$ is equivalent to a subspace of $L_{1}(\mu)^{*}$.

If $\mu$ is not $\sigma$-finite then $L_{1}(\mu)$ contains a subspace isometric with an $l_{1}\left(I_{u}\right)$ and $M(\mu)$ contains a subspace equivalent to $m_{0}\left(I_{u}\right)$; by Theorems 8,9 , and 1 , $L_{1}(\mu)$ is not sc and $M(\mu)$ is not sm.

Theorem 12. $l_{1}(I)$ is sc. $C(K(I))$ is sm.

Klee proved the first part of this in (A1.8); we gave another proof by Theorem 5 when proving $c_{0}(I)$ smooth. Klee (A1.9) also has the result that $M(\mu)$ is sc when $\mu$ is finite, except for an omission in the hypotheses of A1.9; the second part of Theorem 12 follows at once from Theorem 11; when a 
product of Lebesgue measures is introduced in $K(I), C(K(I))$ is isometrically embedded in $M(\mu)$, where $\mu(K(I))=1$. A third proof that $l_{1}(I)$ is sc can also be given by observing that the mapping which sends $\left\{t_{i}\right\}$ in $l_{1}(I)$ to $\sum_{i} t_{i} x_{i}$, where $x_{i}$ is the $i$ th coordinate function on $K(I)$, is an isometry of $l_{1}(I)$ into $C(K(I))$.

By the device of embedding $m_{0}(I)$, Theorem 11 can be completed by

CoROLlaRy. If $M(\mu)$ is not finite-dimensional; that is, if there exist infinitely many disjoint sets of positive $\mu$-measure, then $M(\mu)$ is not sm.

It is not now known whether every sm space must have an sc conjugate, or whether every $L_{1}(\mu)$ must be sc. The most accessible space to investigate further seems to be an uncountable product measure of Lebesgue measures.

From Theorem 5 and the negative results of this section we derive also

COROLLARY. There can be no linear mapping of any $l_{p}(I)$ or $L_{p}(\mu)$ with $p>1$ into a dense subspace of $l_{1}(I), I$ uncountable, or a dense subspace of $m_{0}(I)$, I infinite, or of $M(\mu)$, if $M(\mu)$ is not finite-dimensional.

4. Kakutani's and Michael's extension theorems. Kakutani [3] proved that if $H$ is a locally separable, closed subset of a metric space $E$, and if $C(H)$ and $C(E)$ are the Banach spaces of bounded continuous real functions of $H$ and on $E$, respectively, then there is for each $x$ in $C(H)$ an element $T x$ in $C(E)$, with $T x(h)=x(h)$ for all $h$ in $H$, such that $T$ is non-negative, linear isometry of $C(H)$ into $C(E)$. Michael [5, Theorem 7.1], improved this: If $H$ is a closed subset of a metric space $E$ and if $C(H, Y)$ and $C(E, Y)$ are the spaces of bounded continuous functions into the locally convex linear topological space $Y$, then for each $x$ in $C(H, Y)$ there is a $T x$ in $C(E, Y)$, with $T x(h)=x(h)$ for all $h$ in $H$, such that (a) $T$ is linear and one-to-one from $C(H, Y)$ into $C(E, Y)$, (b) the set of values of $T x$ is in the closed convex hull of the set of values of $x$, and (c) $T$ is continuous in any of three natural topologies in the function space, for example in the topology of uniform convergence.

Obviously whenever there is a retraction $r$ of a topological space $E$ onto a closed subset $H$, the definition $T x(a)=x(r(a))$ for all $a$ in $E$ gives a $T$ with the desired properties. Michael [5] and Klee [4] give an example of a compact Hausdorff space $E$ and a closed subset $H$ which is not a neighborhood retract of $E$; their example is $E=K(I), H=H_{p}(I)$, where $I$ has the cardinal number of the continuum. Their proof involves separability, and says nothing final about the possibility of an extension theorem.

Whenever $I$ is countable, $K(I)$ is a separable metrizable space, and Kakutani's theorem applies to give an embedding-by-extension of $C\left(H_{p}\right)$ into $C(E)$ for every neighborhood $E$ of $H_{p}$ in $K(I)$. When $I$ is not countable no such embedding by extension can occur.

THEOREM 13. If $I$ is uncountable and if $E$ is a neighborhood of $H_{p}(I)$ in 
$K(I)$, then there is no linear mapping of $C\left(H_{p}\right)$ into $C(E)$ which is a simultaneous extension of all elements of $H_{p}$.

COROLlary. If $I$ is uncountable, $H_{p}$ is not a retract of $K$.

The proof to be given is direct and proves somewhat more. If $p>1$, let $1 / p+1 / q=1$; then $H_{p}=H_{p}(I)$ can be regarded as the unit sphere of $l_{p}(I)$ in its weak topology, and there is a natural isometry $Q_{p}$ of $l_{q}(I)$ into $C\left(H_{p}(I)\right)$. Similarly, $H_{1}(I)$ is the unit sphere of $l_{1}(I)$ in its weak*-topology when regarded as the conjugate space of $c_{0}(I)$, and there is a natural isometry $Q_{1}$ of $c_{0}(I)$ into $C\left(H_{1}(I)\right)$. Then Theorem 11 follows directly from

Theorem 14. If $I$ is uncountable and $p \geqq 1$, let $H=H_{p}(I)$; let $L=Q_{p}\left(l_{q}(I)\right)$ if $p>1, L=Q_{1}\left(c_{0}(I)\right)$ if $p=1$; and let $E$ be a neighborhood of $H$ in $K=K(I)$. Then there is no linear function $T$ from $L$ into $C(E)$ such that, for each $x$ in $L$, $T x$ is an extension of $x$.

The case $p=1$ of Theorem 12 appeared when I was trying to prove $c_{0}(I)$ sc by embedding it in $C(K(I))$; Theorem 12 shows that this method must fail, though Theorem 8 , whose proof was discovered much later, says $c_{0}(I)$ is sc.

Proof of Theorem 14. The functions $\phi_{i}$ defined by $\phi_{i}(i)=1, \phi_{i}(j)=0$ if $j \neq i$, all belong to $c_{0}(I)$ and to all $l_{q}(I)$. Using the appropriate $Q_{p}$, let $\Phi_{i}=Q_{p} \phi_{i}$, $i \in I$; then $\Phi_{i} \in L$ and $\Phi_{i}(x)=x(i)$ for each $x$ in $H=H_{p}$ and each $i$ in $I$.

Suppose $T$ is additive from $L$ into $C(E)$, where $E$ is open and contains $H$. Let $x_{i}=\phi_{i}$, but think of $x_{i}$ as an element of $H$. Then $T \phi_{i}\left(x_{i}\right)=\Phi_{i}\left(x_{i}\right)=1$, so there is a neighborhood $U_{i}$ of $x_{i}$ in $E$ such that $\left|T \phi_{i}(x)-1\right|<1 / 3$ if $x \in U_{i}$. $U_{i}$ is determined by a finite set $\sigma_{i} C I$ and a positive number $\delta_{i}$ which we may also assume to be $<1 / 3: U_{i}=\left\{x|| x(j)-x_{i}(j) \mid<\delta_{i}\right.$, for all $j$ in $\left.\sigma_{i}\right\}$.

Now suppose that $i_{1}, \cdots, i_{n}$ are distinct points of $I$. If there is a point $x$ in $\bigcap_{j \leqq n} U_{i_{j}}$, then

$$
\begin{aligned}
\left|\left[T\left(\sum_{j \leqq n} \Phi_{i_{j}}\right)\right](x)-n\right| & =\left|\left[\sum_{j \leqq n} T \Phi_{i_{j}}\right](x)-n\right|=\left|\sum_{j \leqq n}\left(T \Phi_{i_{j}}(x)-1\right)\right| \\
& \leqq \sum_{j \leqq n}\left|T \Phi_{i_{j}}(x)-1\right|<n / 3,
\end{aligned}
$$

so

$$
\left[T\left(\sum_{j \leqq n} \Phi_{i_{j}}\right)\right](x)>2 n / 3
$$

while

$$
\left\|\sum_{j \leqq n} \Phi_{i_{j}}\right\|_{C\left(H_{p}\right)}= \begin{cases}\left\|\sum_{j \leqq n} \phi_{i_{j}}\right\|_{c_{0}(I)}=1 & \text { if } p=1, \\ \left\|\sum_{j \leqq n} \phi_{i_{j}}\right\|_{l_{q}(I)}=n^{1 / q} & \text { if } p>1 .\end{cases}
$$


Hence, if $T$ is linear, that is additive and bounded, and if $x \in \bigcap_{j \leqq n} U_{i_{j}}$, then

$$
2 n / 3 \leqq\|T\| \quad \text { if } p=1 \text { and } 2 n / 3 \leqq n^{1 / q}\|T\| \quad \text { if } \quad p>1 \text {, }
$$

so, setting $P=(3\|T\| / 2)^{p}$, we have

$$
n \leqq P \quad \text { if } \quad x \in \bigcap_{j \leqq n} U_{i_{j}} .
$$

But $\bigcap_{j \leqq n} U_{i j}$ is empty if and only if some $i_{j}$ is in some $\sigma_{i_{k}}$ and $i_{j} \neq i_{k}$. Therefore

If $n>P$ and $i_{1}, \cdots, i_{n}$ are distinct points of $I$, there is at least one $i_{j}$ in $\bigcup_{k \neq j} \sigma_{i_{k}}$.

With this knowledge we define a sequence $\left\{i_{j}\right\}$ of points of $I$ and an increasing sequence $\left\{m_{j}\right\}$ of integers as follows: Choose $i_{1}, \cdots, i_{m_{1}}$ so that $i_{j} \notin \sigma_{i_{k}}$ if $j, k \leqq m_{1}$ and $j \neq k$, and choose $m_{1}$ maximal under this condidition; then $m_{1} \leqq P$ and for every $i$ not in $M_{1}=\bigcup_{j \leqq m_{1}} \sigma_{i_{j}}$ at least one $i_{j}$ is in $\sigma_{i}$. Then choose $m_{2}$ maximal under the condition that for $m_{1}<k \leqq m_{2}$ the point $i_{k} \notin \sigma_{i_{j}}$ if $j \neq k$ and $j \leqq m_{2}$. Then $m_{2}-m_{1} \leqq P$, and when $i \notin M_{2}=\bigcup_{k \leqq m_{2}} \sigma_{i_{k}}$, at least one $i_{k}, m_{1}<k \leqq m_{2}$, lies in $\sigma_{i}$.

Continue this process by induction and let $M=\mathrm{U}_{n} M_{n}$. Then if $i \notin M$, there is for each $n$ a $k_{n}$ with $m_{n-1}<k_{n} \leqq m_{n}$ such that $i_{k_{n}} \in \sigma_{i}$. If there were such an $i$ this would make the finite set $\sigma_{i}$ infinite; but $M$ is countable and $I$ is not, so we have reached a contradiction. This shows that no such $T$ as we had assumed can exist.

\section{BIBLIOGRAPHY}

1. L. Alaoglu and G. Birkhoff, General ergodic theorems, Ann. of Math. (2) vol. 41 (1940) pp. 293-309.

2. J. A. Clarkson, Uniformly convex spaces, Trans. Amer. Math. Soc. vol. 40 (1936) pp. 396-414.

3. S. Kakutani, Simultaneous extension of continuous functions considered as a positive linear operation, Jap. J. Math. vol. 17 (1940) pp. 1-4.

4. V. L. Klee, Convex bodies and periodic homeomorphisms in Hilbert space, Trans. Amer. Math. Soc. vol. 74 (1953) pp. 10-43.

5. E. Michael, Some extension theorems for continuous functions, Pacific Journal of Mathematics vol. 3 (1953) pp. 789-806.

6. R. S. Phillips, On weakly compact subsets of a Banach space, Amer. J. Math. vol. 65 (1943) pp. 108-136.

UNIVERSITY OF ILLINOIS,

URBANA, ILL. 